\title{
Concentration dependent different action of progesterone on the order, dynamics and hydration states of the head group of dipalmitoyl-phosphatidylcholine membrane
}

\author{
Filiz Korkmaz $^{\mathrm{a}}$, Halil Kirbıyık ${ }^{\mathrm{b}}$ and Feride Severcan ${ }^{\mathrm{c}, *}$ \\ ${ }^{a}$ Materials Science Engineering, Attlum University, Ankara 06836, Turkey \\ ${ }^{\mathrm{b}}$ Department of Physics, Middle East Technical University, Ankara 06531, Turkey \\ ${ }^{\mathrm{c}}$ Department of Biology, Middle East Technical University, Ankara 06531, Turkey
}

\begin{abstract}
Interactions of progesterone with zwitterionic dipalmitoyl phosphatidylcholine (DPPC) multilamellar liposomes (MLVs) were investigated as a function of progesterone concentration at selected temperatures monitoring both the gel and liquid crystalline phase, by using Fourier Transform Infrared spectroscopy (FTIR). It has been show that the effect of progesterone on membrane dynamics is dependent on progesterone concentration. At $1 \mathrm{~mol} \%$, which is close to physiological level, progesterone behaves differently. At this concentration the decrease in dynamics is more noticeable. Additionally a dramatic decrease in the strength of hydrogen bonding in the interfacial region of the bilayer is also observed. When concentration increases up to $12 \mathrm{~mol} \%$ opposite behaviour is observed at all interactions. Above $12 \mathrm{~mol} \%$, progesterone-DPPC interactions shows almost linear plot.
\end{abstract}

Keywords: DPPC liposome, progesterone, FTIR, membrane fluidity, lipid order

\section{Introduction}

Besides being secreted in small amounts naturally by the corpus luteum in human body, progesterone is also used in drugs at high concentrations in hormone replacement therapy, osteoporosis, and the treatment of cysts [1-3] and these drugs produce progesterone plasma concentrations beyond physiological levels of women with normal menstrual cycles. At such high concentrations steroids also show nongenomic action by perturbing lipid-lipid interactions besides the well-known genomic action. Despite their importance, the studies on progesterone-membrane interaction are very limited [4-8]. These spectroscopic and calorimetric studies mainly reported the effect of progesterone on lipid dynamics and location of the drug in membranes. It has to be noted that the findings of these studies are not in agreement with each other. We have recently studied progesterone-zwitterionic dipalmitoyl phosphatidylcholine (DPPC) interaction as a function of temperature by using Fourier Transform Infrared (FTIR), visible spectroscopy, and differential scanning calorimetry (DSC) [9]. In the referenced paper it was shown that progesterone decreases the main phase transition temperature, abolishes the pretransition, broadens the

\footnotetext{
*Corresponding author. Phone: +90 312210 5166; Fax: +90 312210 1289; E-mail: feride@ @etu.edu.tr.
} 
phase transition profile, disorders the system both in gel and liquid crystal phase, increases the dynamics at low concentrations whereas stabilizes the membrane at high concentrations and induces phase separation.

It is important to know the concentration dependent behavior of progesterone in detail, since its dose dependent adverse effects were reported previously [4-6,9]. Therefore, in the current work, the concentration dependent behavior of progesterone on zwitterionic dipalmitoyl phosphatidylcholine (DPPC) multilamellar liposomes using FTIR spectroscopy was studied in detail. In addition to membrane fluidity [9], in the present work, it was also shown that progesterone has different action on lipid order and hydrogen binding capacity of both carbonyl and phosphate head group of lipid membrane depending on progesterol concentrations.

\section{Experimental}

Progesterone and DPPC were purchased from Sigma (St. Louis, MO, USA) and the chemicals were used without further purification $[10,11]$.

\subsection{Preparation of phosphatidylcholine liposomes}

Phospholipid MLVs were prepared for FTIR study according to the procedure reported previously [10,11]. $5 \mathrm{mg}$ of DPPC was dissolved in $0.15 \mathrm{ml}$ chloroform inside an ependorf. In order to obtain uniform distribution of the lipid on the walls of the container, excess chloroform was removed by a stream of nitrogen. Then remaining organic solvent was removed by subjecting the sample to vacuum drying by Spin Vax for 2 hours. The dry films were then hydrated by adding $25 \mu 1$ of $10 \mathrm{mM}$ phosphate buffer ( $\mathrm{pH}$ 7.4). MLVs were formed by vortexing the mixture for $20 \mathrm{~min}$ at $20^{\circ} \mathrm{C}$ above the $T_{\mathrm{m}}$ of DPPC $\left(\sim 62^{\circ} \mathrm{C}\right)$. Progesterone containing liposomes were prepared as reported in Korkmaz and Severcan, 2005 [9]. The required amount of progesterone from stock solution was initially placed inside the sample tube. Then, excess ethanol was removed by a stream of nitrogen, next lipid was added and the procedure mentioned above for the preparation of DPPC liposomes was followed. For FTIR measurements, $20 \mu \mathrm{l}$ of liposomes were mounted between $\mathrm{CaF}_{2}$ windows with $12 \mu \mathrm{m}$ sample thickness.

\subsection{FTIR measurements and spectral analysis}

Spectra were recorded on a BOMEM 157 FTIR spectrophotometer (The Michelson Series, Bomem, Inc. Quebec, Canada) equipped with a DTGS (deuterated triglycine sulfate) detector in the temperature range of $25-60^{\circ} \mathrm{C}$. The temperature was controlled by Graseby Specac digital temperature controller unit and a thermocouple located against the edge of the cell windows. Samples were incubated for $5 \mathrm{~min}$ at each temperature before scanning of the spectrum. Interferograms were averaged for 50 scans at $2 \mathrm{~cm}^{-1}$ resolution.

To get a better resolution of the infrared bands, the water bands were subtracted, leaving a flattened water band at the location around $2125 \mathrm{~cm}^{-1}$. This was done by using Win Bomem Easy Software (Galactic Industries Corporation). The band positions were measured from the center of weight. Averages of the spectra belonging to the same experimental group were calculated by using GRAMS/32 software program. 


\section{Results and discussion}

In this study DPPC MLVs were investigated as a function of progesterone concentration at different temperatures that monitors different membrane phase states such as gel, phase transition, and liquid crystalline state by FTIR spectroscopy. In the analysis of the FTIR spectra, $\mathrm{CH}_{2}$ symmetric and antisymmetric, $\mathrm{C}=\mathrm{O}$ stretching and $\mathrm{PO}_{2}^{-}$double stretching bands were studied. All experiments were repeated three times and averages of the spectral parameters mentioned above were plotted as a function of progesterone concentration. Previously Korkmaz and Severcan [9] showed that the contribution of progesterone to the $\mathrm{C}-\mathrm{H}$ and $\mathrm{C}=\mathrm{O}$ stretching bands is negligible. Therefore the $\mathrm{C}-\mathrm{H}$ and $\mathrm{C}=\mathrm{O}$ stretching bands, in the spectra of DPPC containing progesterone, mainly monitors the lipid functional groups. Figure 1 shows average FTIR spectra of DPPC MLVs in the absence and presence of progesterone in liquid crystalline phase $\left(50^{\circ} \mathrm{C}\right)$. In this figure, the normalized average spectra are presented to see clearly the relative changes in the frequency, signal intensity and bandwidth of the specific bands of interest. However, for the precise determination of the variations, each of the original baselined spectra was used.

Figure 2 shows temperature dependence of the frequency of the $\mathrm{CH}_{2}$ symmetric stretching mode of DPPC MLVs in the absence and presence of varying concentrations of progesterone at three different temperatures. Our previous DSC study showed that with the addition of progesterol mail phase transition temperature of DPPC which is observed at $41^{\circ} \mathrm{C}$ slightly shifts to lower temperatures $\left(1\right.$ to $2^{\circ} \mathrm{C}$, depending on progesterol concentration) [9]. Therefore, the temperatures were chosen as $28^{\circ} \mathrm{C}, 41^{\circ} \mathrm{C}$ and $50^{\circ} \mathrm{C}$ to represent the system in gel phase, main phase transition region and in liquid crystalline phase respectively. It is well known that an increase in the frequency implies a decrease in the order of the system [10]. As seen from the figure, although generally a slight increase at $28^{\circ} \mathrm{C}$ and a negligible variation at $50^{\circ} \mathrm{C}$ in the frequency are observed at $1 \mathrm{~mol} \%$ progesterone, the frequency of the $\mathrm{CH}_{2}$ symmetric stretching band dramatically increases with respect to that of pure DPPC at $41^{\circ} \mathrm{C}$. For other concentrations an increase in the frequency is observed relative to pure DPPC at all temperatures. In the phase transition region $\left(\right.$ at $41^{\circ} \mathrm{C}$ ) a gradual increase in the frequency is observed as progesterone concentration increases. Another interesting thing to note is that, above $12 \mathrm{~mol} \%$ progesterone concentration, almost a linear plot is obtained. These results are in good agreement with a recent spin label ESR study on progesterone-erythrocytes membrane interactions where a decrease in membrane order was also reported at $37^{\circ} \mathrm{C}[8]$.

Figure 3 shows the $\mathrm{CH}_{2}$ antisymmetric stretching bandwidth of DPPC as a function of progesterone concentration. The vibrational bands of $\mathrm{CH}_{2}$ symmetric and antisymmetric stretching mode were suffi-

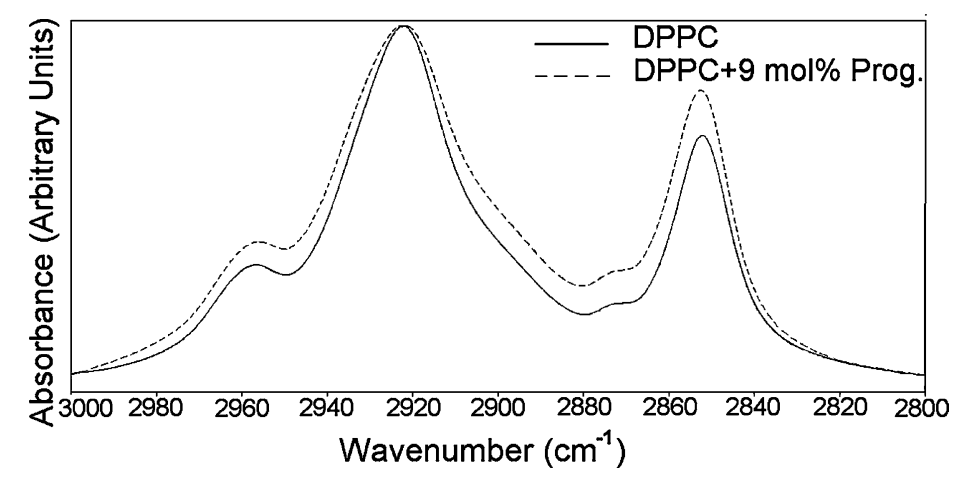

Fig. 1. Infrared spectra of DPPC liposomes in the absence and presence of $9 \mathrm{~mol} \%$ progesterone at $50^{\circ} \mathrm{C}$ in the $3000-2800 \mathrm{~cm}^{-1}$ region. 


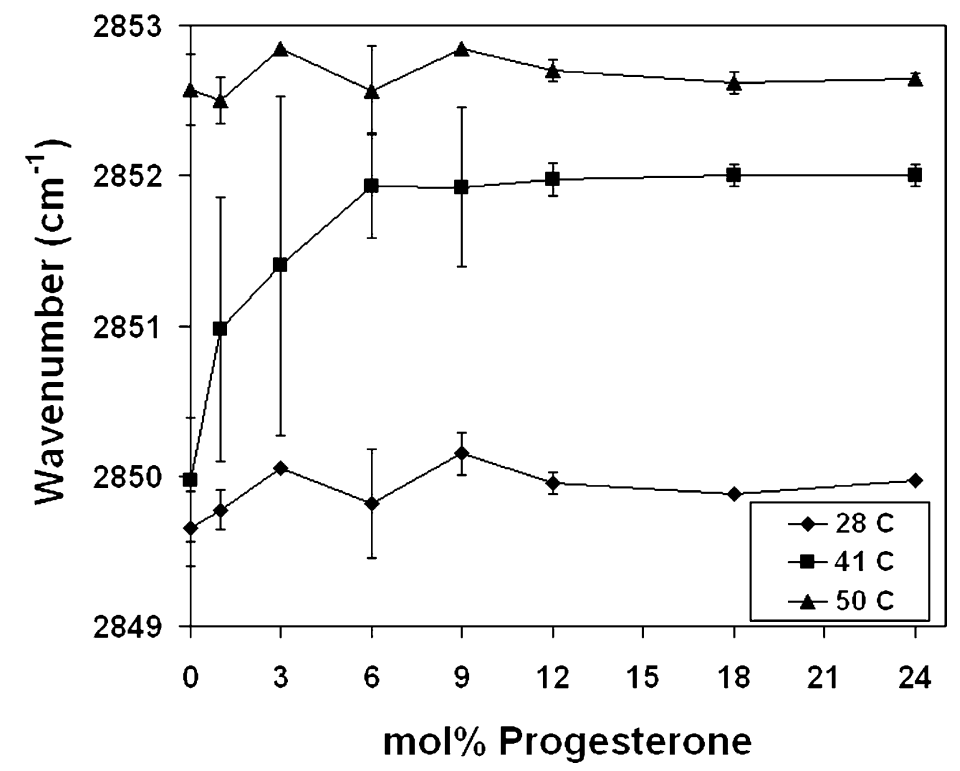

Fig. 2. Progesterone concentration dependent variation in the frequency of the $\mathrm{CH}_{2}$ symmetric stretching modes of DPPC liposomes at different temperatures.

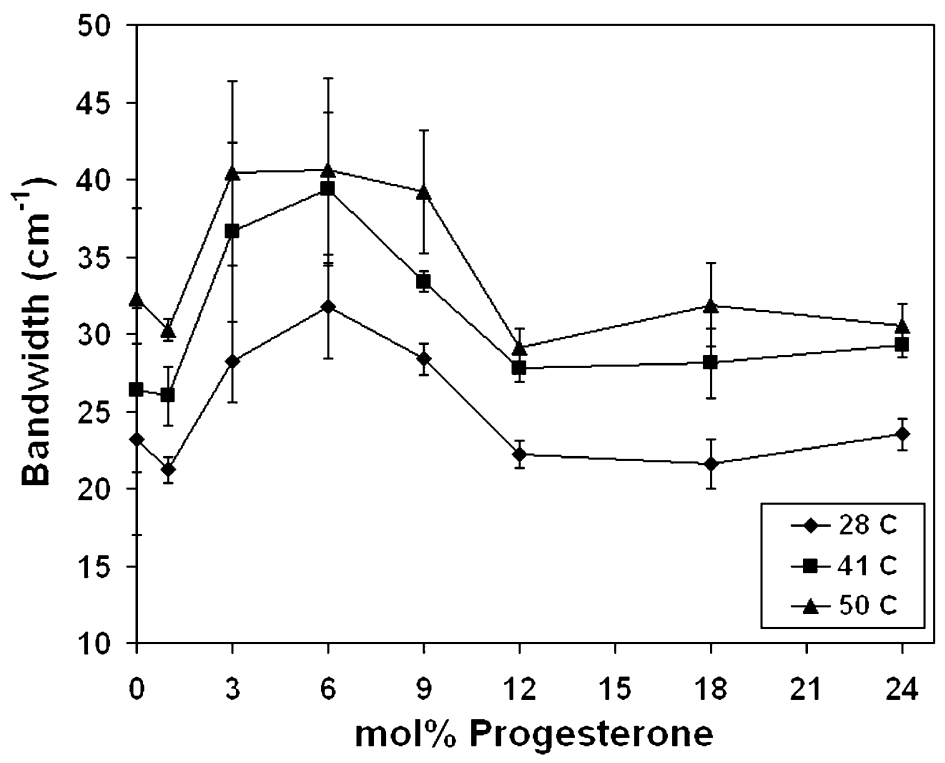

Fig. 3. Progesterone concentration dependence of the bandwidth of $\mathrm{CH}_{2}$ antisymmetric stretching modes of DPPC liposomes at different temperatures.

ciently separated after water subtraction procedure. It was not therefore necessary to use band deconvolution or fit routines to evaluate their bandwidths for relative measurements in this model membrane study, as was also reported by others $[12,13]$. The widths were measured at $50 \%$ of height of the peaks. The variation of the bandwidth gives information about the dynamics of the system. An increase in bandwidth is the indication of an increase in dynamics [9-11]. It is evident from Fig. 3 that, at all phases, 


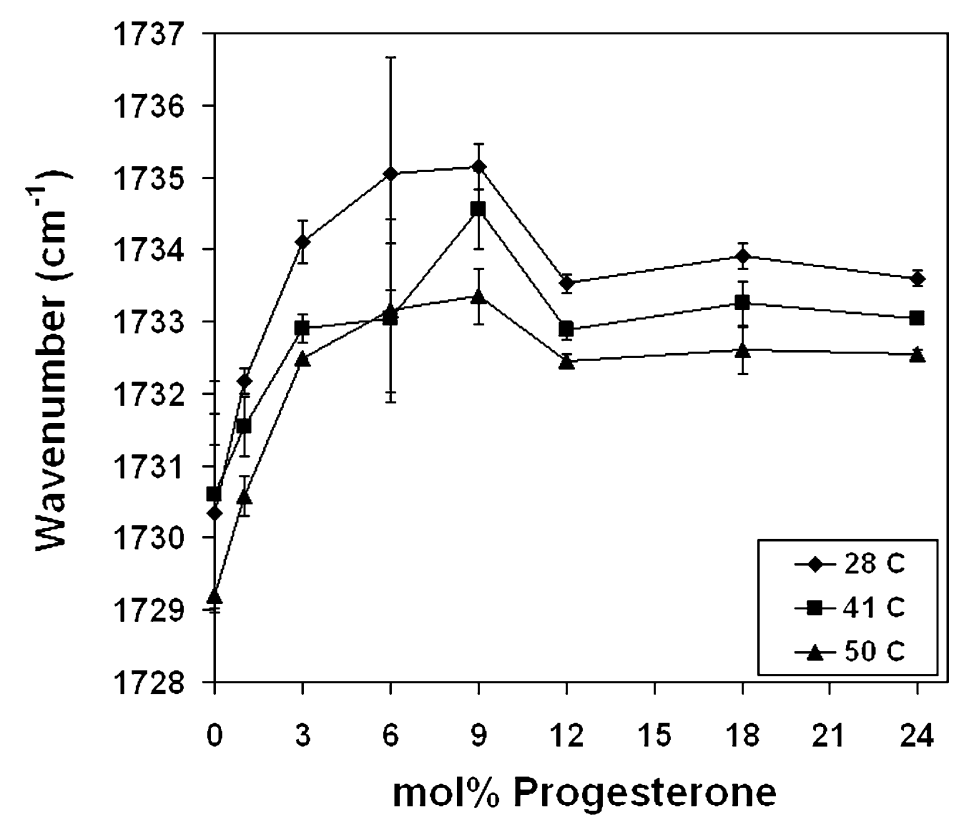

Fig. 4. Progesterone concentration dependence of frequency changes of the $\mathrm{C}=\mathrm{O}$ stretching mode for DPPC at different temperatures.

progesterone shows different effects on the fluidity of DPPC MLVs with varying progesterone concentration. At very low (1 mol\%) and high concentrations (12, 18 and $24 \mathrm{~mol} \%)$, progesterone stabilizes the system, whereas at low concentrations $(3,6$ and $9 \mathrm{~mol} \%)$, it increases the membrane dynamics. Interestingly the bandwidth values also give almost a linear plot above $12 \mathrm{~mol} \%$ progesterone concentration similar to the behavior of the $\mathrm{CH}_{2}$ symmetric stretching frequency depicted in Fig. 2.

In order to examine the interaction of progesterone with glycerol backbone near the head group of phospholipids in interfacial region, the $\mathrm{C}=\mathrm{O}$ stretching band was analyzed [10]. The temperature dependence of the frequency of this band is shown in Fig. 4. According to the empirical rules a decrease in the frequency implies either the strengthening of existing hydrogen bonding or formation of new hydrogen bonding between the components $[11,14]$. In the current study the frequency shifts to higher values for all samples containing progesterone, indicating that there is no evidence of hydrogen bonding. It is possible that progesterone displaces some $\mathrm{H}_{2} \mathrm{O}$ molecules from the interfacial region resulting in an increase in the number of free carbonyl groups. Again almost a linear plot is obtained for progesterone concentrations above $12 \mathrm{~mol} \%$.

The interaction between progesterone and head group of DPPC liposomes was monitored by $\mathrm{PO}_{2}^{-}$ antisymmetric double stretching band, which is located at $1230 \mathrm{~cm}^{-1}$. Figure 5 shows progesteroneconcentration dependent variation of the frequency of $\mathrm{PO}_{2}^{-}$antisymmetric double stretching band of DPPC liposomes at different temperatures. As seen from the figure, the frequency shifts firstly to higher values at $1 \mathrm{~mol} \%$ progesterone and then at $3 \mathrm{~mol} \%$ progesterone, it decreases to even much lower values than those of DPPC. It then starts to increase with the increase in progesterone concentration and finally shows a linear behavior at and above $12 \mathrm{~mol} \%$ progesterone. These results imply that only 3 and $6 \mathrm{~mol} \%$ progesterone cause strengthening in hydrogen bonding between the head group of liposomes either with the $\mathrm{C}-\mathrm{H}$ group of progesterone or with water molecules in the vicinity $[11,15]$. On the other hand, an increase in the wave number of oscillation observed at lower and higher progesterone concentrations 


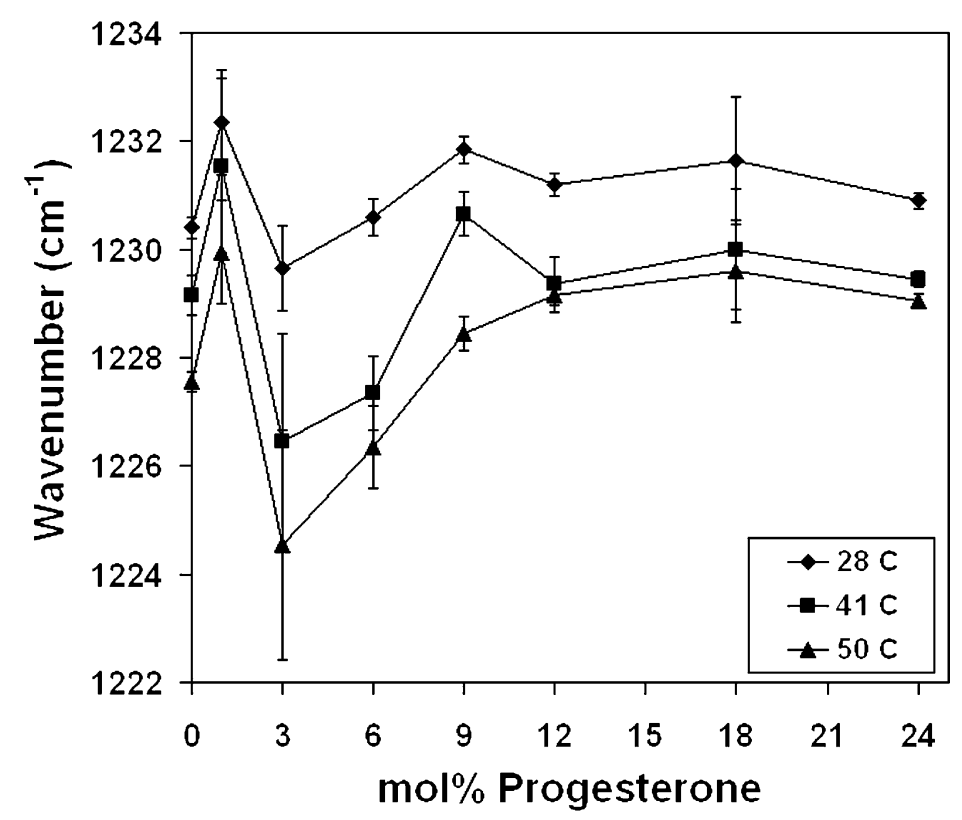

Fig. 5. Progesterone concentration dependence of frequency changes of the $\mathrm{PO}_{2}^{-}$antisymmetric double stretching modes of DPPC liposomes at different temperatures.

implies an increase in dehydration. This effect is more conspicuous in the liquid crystalline phase and especially at $1 \mathrm{~mol} \%$ progesterone. Similarly a linear plot is obtained for concentrations above $12 \mathrm{~mol} \%$.

A particular attention has been paid to distinguish the structural parameters describing molecular order, and motion parameters such as bandwidth describing molecular dynamics as was also suggested by others $[10,11,16]$. There is limited number of studies done about the effect of progesterone on membrane dynamics [6-8]. It must be stated that findings in these studies are not in agreement with each other. Whiting et al. [6], using steady state fluorescence polarization, reported that high concentrations of progesterone ( $>10 \mathrm{~mol} \%)$ decreases lipid fluidity in both egg yolk phosphatidylcholine and natural membranes. Liang et al. [7] also reported the same effect on membrane dynamics in liposomes and in cells using fluorescence spectroscopy. In contrast, Tsuda et al. [8] recently reported progesteroneinduced increase in membrane fluidity in erythrocytes membranes. Results of the current study clearly show that the effect of progesterone on membrane dynamics depend on progesterone concentration. A dramatic increase in the dynamics of DPPC membranes is observed at low concentrations of progesterone (3, 6 and $9 \mathrm{~mol} \%$ ) both in the gel and liquid crystalline phases (Fig. 3). On the other hand, a decrease in dynamics is observed at lower and higher concentrations. This decrease is more noticeable at $1 \mathrm{~mol} \%$ progesterone for which a dramatic decrease in the strength of hydrogen bonding in the interfacial region of the bilayer is also observed.

Although the physiological level of progesterone is very low (around $1 \mathrm{~mol} \%$ ), it is administered at doses as high as $800 \mathrm{mg} / \mathrm{d}$ in various diseases $[17,18]$. It was previously reported that the adverse effects of drugs may be dose dependent $[19,20]$. It is therefore vital to know precisely the concentration dependent behavior of drugs on membrane fluidity and other physical parameters.

In conclusion, in the present study the concentration dependent action of progesterone on lipid order and dynamics has been investigated in detail. Besides hydration states of the head and the region near to aqueous region of zwitterionic DPPC MLVs at temperatures monitoring the gel and liquid crystalline 
phase and phase transition region were also studied. The results revealed that progesterone behaves differently at $1 \mathrm{~mol} \%$, which is close to the physiological level. For higher progesterone concentrations up to $12 \mathrm{~mol} \%$, an opposite behavior is observed in the interactions including membrane fluidity. The considered effects of progesterone on frequencies and bandwidth of DPPC membranes reach a saturation level such that no further influence is seen beyond $12 \mathrm{~mol} \%$. Thus it can be inferred that 1 and $12 \mathrm{~mol} \%$ are critical concentrations for administration of progesterone as drug. Membrane fluidity is one of the important parameters for drugs to reach at the specific target places. Therefore, it is believed that the results of the present study will elucidate the dose dependent different actions of progesterone reported in literature.

\section{References}

[1] J.R. Lee, Medical Hypothesis 35 (1991), 316.

[2] C. Walter, Jr., M.D. Barnes, The American J. of Surg. 129 (1975), 324.

[3] P. Kenemans and A. Bosman, Best Practice \& Research Clinical Endocrinology \& Metabolism 17 (2003), 123.

[4] J.C. Carlson, M.Y. Gruber and J.E. Thompson, Endocrinology 113 (1983), 190.

[5] A. Sanchez-Bueno, S. Watanabe, M.J. Sancho and T. Saito, J. Steroid Biochem. Molec. Biol. 38 (1990), 173.

[6] K.P. Whiting, C.J. Restall and P.F. Brain, Life Sciences 67 (2000), 743.

[7] Y. Liang, S. Belford, F. Tang, L. Proka3i, J.W. Simpkins and J.A. Hughes, Brain Research Bulletin 54 (2001), 661.

[8] K. Tsuda, Y. Kinoshita and I. Nishio, American J. of Hypert. 15 (2002), 702.

[9] F. Korkmaz and F. Severcan, Arch. Biochem. Biophys. 440 (2005),141.

[10] N. Toyran and F. Severcan, Chem. Phys. Lipids 123 (2003), 165.

[11] F. Severcan, I. Şahin and N. Kazanc1, Biochim. Biophys. Acta 1668 (2005), 215.

[12] N. Toyran and F. Severcan, Spectroscopy - Int. J. 16 (2002), 399.

[13] H.C. Chen, R. Mendelsohn, M.E. Rerek and D.J. Moore, Biochim. Biophys. Acta 1468 (2000), 293.

[14] U.P. Frengeli and H.H. Günthard, Biochim. Biophys. Acta 450 (1976), 101.

[15] H.L. Casal, H.H. Mantsch, F. Paltauf and H. Hauser, Biochim. Biophys. Acta 919 (1987), 275.

[16] G. Van Ginkel, H. van Langen and Y.K. Levine, Biochimie 71 (1989), 23.

[17] I.F. Godsland, Fertility and Sterility 75 (2001), 898.

[18] A. Wihlback, S. Nyberg, T. Backstrom, M. Bixo and I. Sundstrom-Poromaa, Psychoneuroendocrinology 30 (2005), 38.

[19] B. Turan, S. Bayarı, C. Balçık, F. Severcan and N. Akkaş, Biometals 13 (2000), 113.

[20] G.R. De Lima, G. Facina, J.Y. Shida, M.B.C. Chein, P. Tanaka, R.C. Dardes, V.C. Jordan and L.H. Gebrim, Eur. J. Cancer 39 (2003), 891 . 


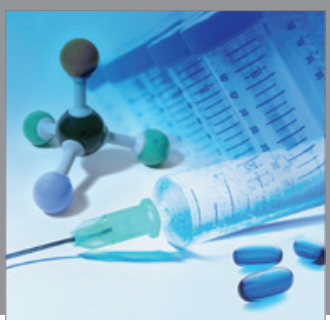

International Journal of

Medicinal Chemistry

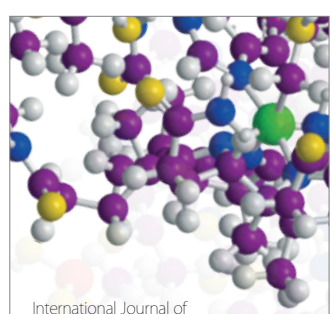

Carbohydrate Chemistry

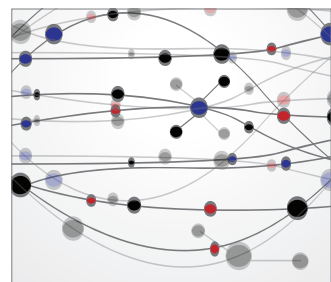

The Scientific World Journal
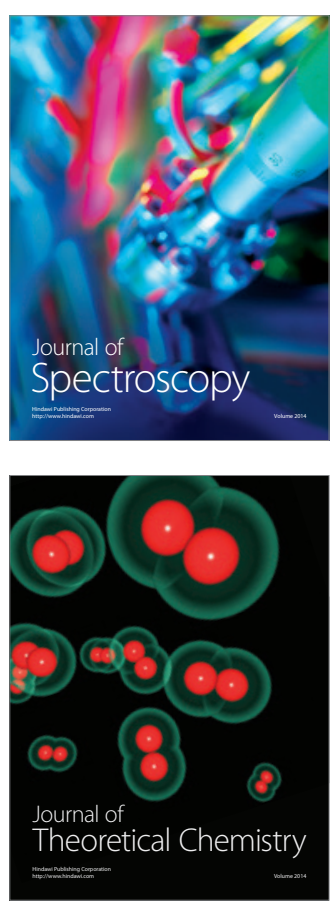
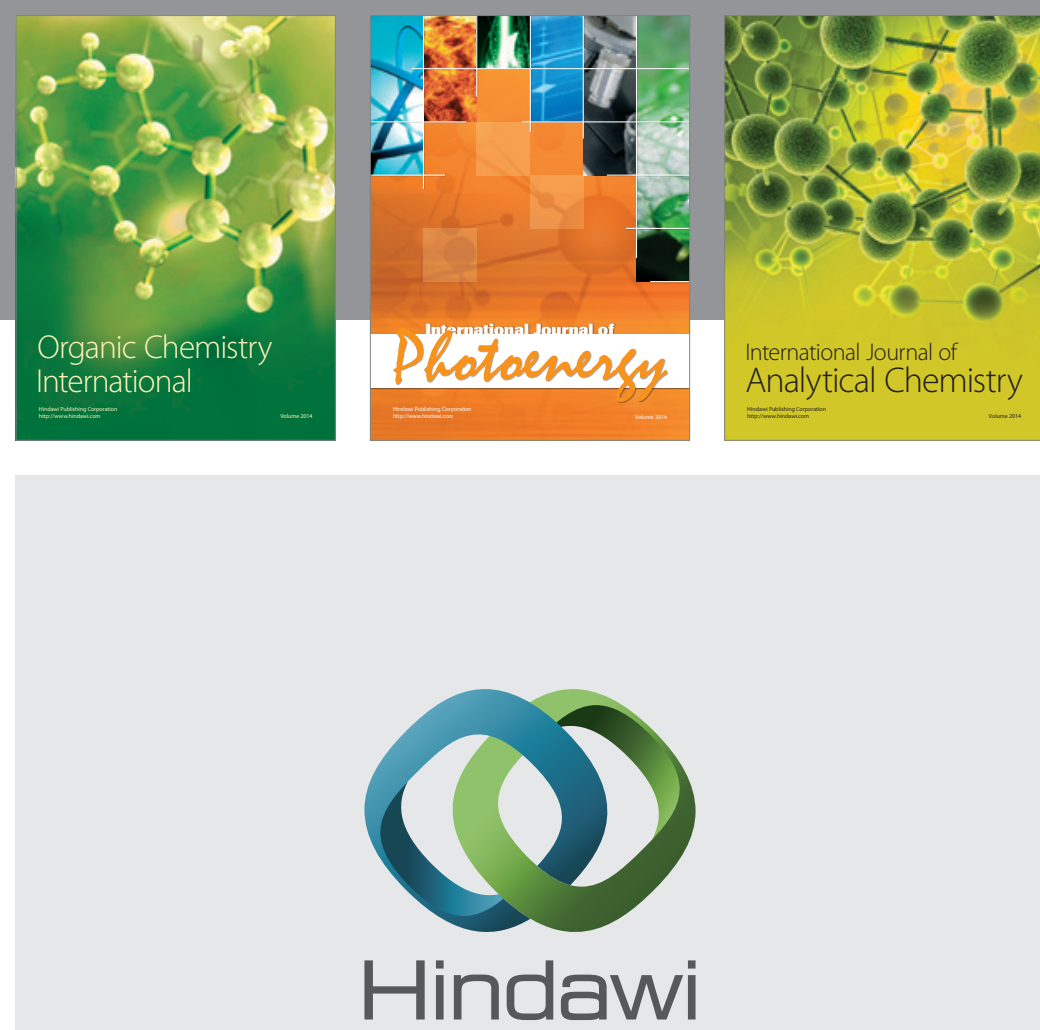

Submit your manuscripts at

http://www.hindawi.com
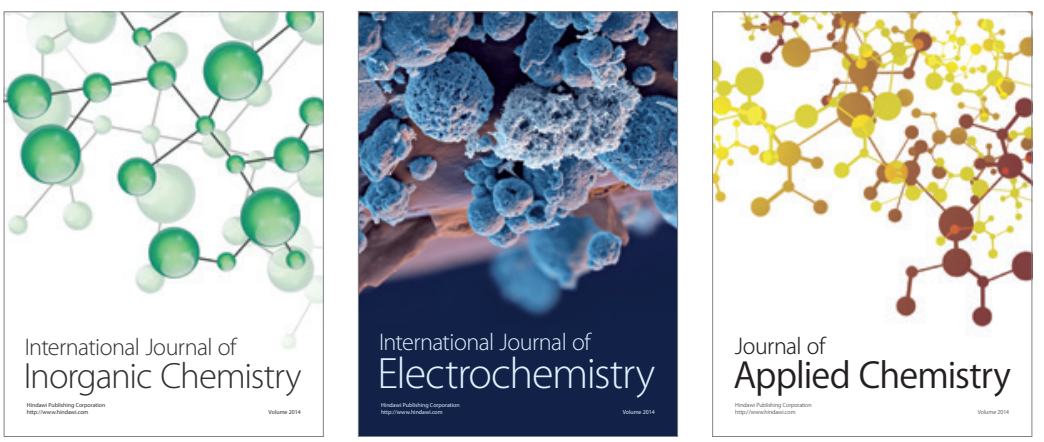

Journal of

Applied Chemistry
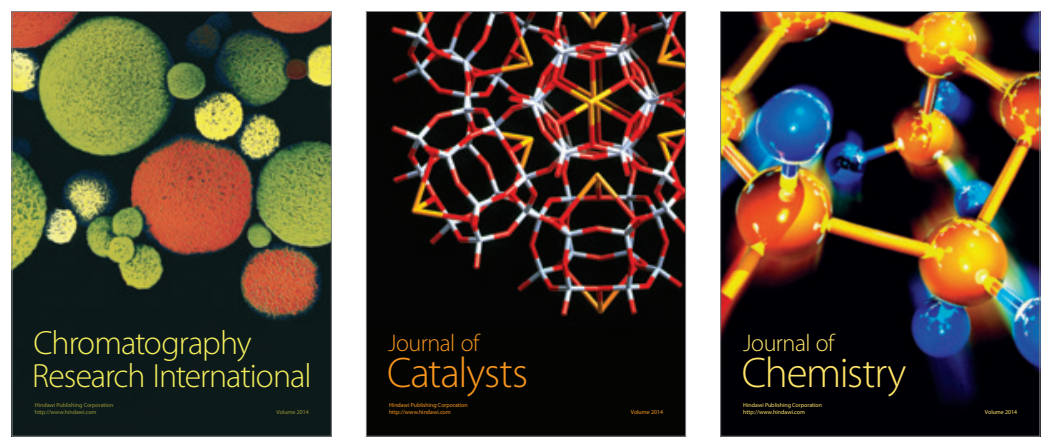
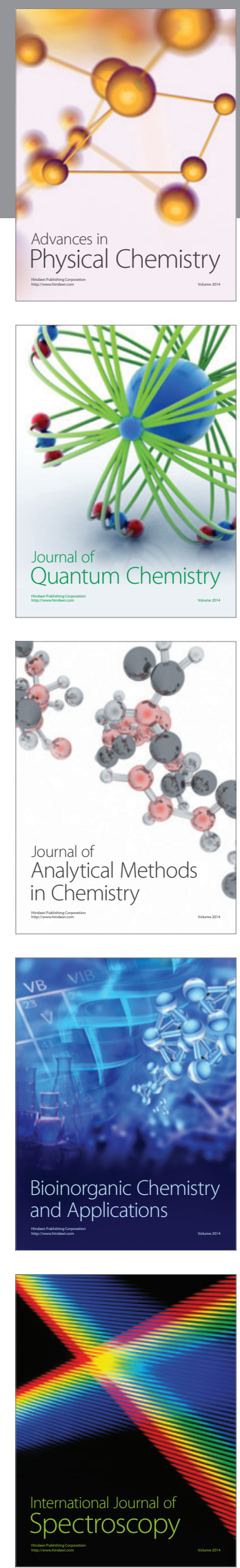